\title{
Alterations in the expression of genes for tubulin and stress in two Arabidopsis auxin mutants during simulated microgravity
}

\author{
P. Tassone', A. Fortunati', A. Saba'2, G. Pani², M.A. Meloni², P. Pippia'², F. Migliaccio'* \\ ' Institute of Agro-environmental and Forest Biology - National Research Council; via Salaria Km 29.300, \\ Monterotondo (Rome), Italy \\ ${ }^{2}$ Dipartimento di Scienze Fisiologiche, Biochimiche e Cellulari - University of Sassari, via Muroni 25 Sassari, Italy \\ * fernando.migliaccio@ibaf.cnr.it
}

KEY WORDS: microgravity, tubulin, auxin, genes expression.

\begin{abstract}
The effect of microgravity on the expression of genes for tubulin, stress and aging in Arabidopsis seedlings from wild-type, and the auxinic mutants auxl and eirlpin2 is reported. Simulated microgravity was produced through the Random Positioning Machine (RPM). The results showed that the tubulin genes TUA4, TUA6 and TUB2, in plants subjected to $\mu \mathrm{g}$, showed a peak of expression at $3 \mathrm{~h}$ in the wild-type, whereas in the mutants it was delayed to $6 h$. The microgravity treatment tested on the expression of stress genes FeSOD1 and CAT3 showed only a significant increase of expression for FeSOD1. The gene involved in senescence SAG12 showed increased expression in the wild-type starting at $3 \mathrm{~h}$, whereas in the mutant a significant increase was seen only at $24 \mathrm{~h}$. From the collected data we conclude that auxin transport and action should be involved in tubulin synthesis, stress, and the senescence process.
\end{abstract}

\section{Introduction}

$\alpha$-TUBULIN (TUA) and $\beta$-TUBULIN (TUB) proteins induce the synthesis of cortical microtubules (MTs), which continuously reorganize the cytoskeleton in response to developmental and environmental cues [1]. MTs play a central role in several processes including plant response to gravity [2]. In plant cells, MT arrays respond, changing their orientation, to different external signals, such as light, mechanical pressure, wounding [3], clinorotation [4], and for this reason Baluska and Hasenstein (1997) [5] suggested that a possible sensor of gravitropism could be the cytoskeleton. In addition, plant root growth patterns are highly affected by the absence of gravity [6-8]. The phyto-hormone auxin influences multiple aspects of growth and differentiation in plants. The hormone is also involved in the gravitropic response through a differential redistribution in roots and shoots, allowing a reorientation of the growth. A large family of proteins mediates auxin transport and redistribution. It was shown, in roots, that mutants in the auxin transport genes, such as aux $I$ and eirl/pin2, do not correctly respond to gravi-stimulation, suggesting a key role played by auxin transport in the gravitropic response [9].

In this work we present preliminary results of the effect caused by a short time exposure of Arabidopsis seedlings to simulated microgravity (24h), on the expression profile of: genes related to cytoskeleton formation, like some $\alpha$ and $\beta$ tubulin isoforms (TUA4, TUA6 and TUB2); genes related to oxidative stress response, i.e. catalase (CAT3), iron superoxidodismutase (FeSODI), and genes activated by senescence such as $S A G 12$.

\section{Materials and methods}

Plant and growth conditions

Seeds of Arabidopsis thaliana plants, ecotype Ler, and mutants aux / and eir I/pin2, after sterilization, were plated on Petri dishes, on a growth medium made up of $1.5 \%$ agar, $1 \%$ sucrose, and $0.5 \times \mathrm{MS}$ salts.

\section{Simulated microgravity treatment}

To simulate microgravity conditions, Arabidopsis 14-d-old seedlings were mounted on the RPM (3D clinostat) for a period of $24 \mathrm{~h}$. RPM speed rotation was set at $60^{\circ}$ s. Treatments were done in a controlled environment. The facility is located at the University of Sassari, Italy. The experiments were repeated twice. For each ecotype, three dishes were applied on the RPM, with at least 30 seedlings each.

\section{Quantitative real time $q P C R$ analysis}

Total RNA was extracted from $50 \mathrm{mg}$ of each sample using the TRIZOL reagent (Invitrogen, CA, USA). Two $\mu \mathrm{g}$ of total RNA was reverse transcribed using the Super Script III (Invitrogen) to synthesize the first-strand cDNA which was used for the polymerase chain reaction (PCR). Real time quantitative polymerase chain reactions were performed using a Mx3000P ${ }^{\circledR}$ (Stratagene, CA, USA) system. The reported data were collected through a 
quantitative real time analysis, 3,6 and $24 \mathrm{~h}$ after the RPM. Relative amounts were normalized with respect to I8S ribosomal expression level, as log2 of the relative mRNA level, calibrated to the not-treated control plants $(=0) . \log 2<0$ represents a down-regulation, and $\log 2>0$ represents an up-regulation of gene expression.

\section{Results}

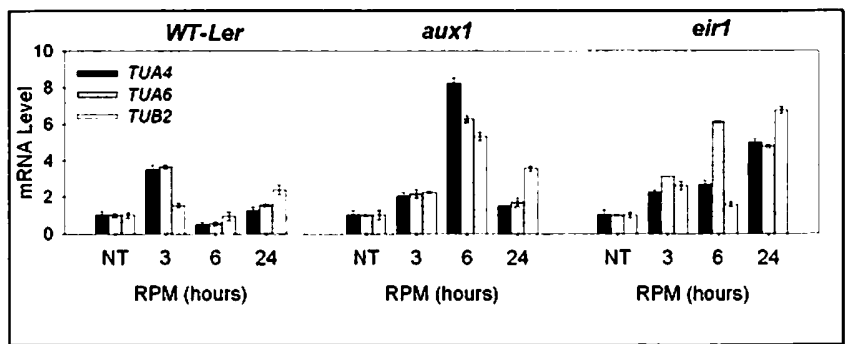

Figure 1. Simulated Microgravity affects the expression of tubulin gene in Arabidopsis plants. The expression profile of $\alpha$-TUBULIN (TUA3 -TUA4) and $\beta$-TUBULIN (TUB2), are shown in wild type (WT-Ler) together with that of two Arabidopsis auxin transport mutants: aux 1, and eirl/pin2

In the reported experiments the effect of microgravity, produced through the RPM, on the expression of the genes TUA4,TUA6 and TUB2 was analyzed. The gene are encoding respectively for $\alpha$ tubulin 4 and 6 and $\beta$ tubulin 2. Their expression was tested in wild-type plant and in two auxin mutants, i.e. aux / and eir//pin2, the first considered to be a facilitator of auxin influx into cells, the second considered a facilitator of the efflux. Sampling was at 3,6 and $24 \mathrm{~h}$ on RPM and in $1 \mathrm{~g}$ controls. In the graph (fig. 1), the data show the effect of different time lapses of microgravity exposure

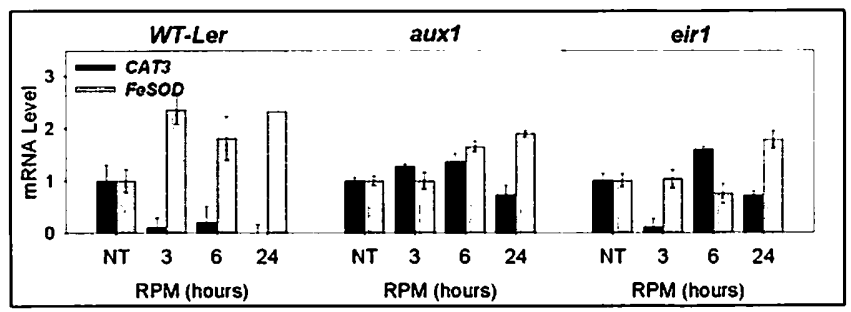

Figure 2. Simulated Microgravity affect the expression of stress gene in Arabidopsis plants. The expression of two genes: CAT3 and FeSODI in wild-type Ler, auxI and eirl/pin2 plants

on the whole Arabidopsis tissue. The results showed a peak of expression of TUA4 and TUA6 after $3 \mathrm{~h}$ of microgravity, in the wild-type Ler, whereas in oux / and eir //pin2 the peak was moved to $6 \mathrm{~h}$. Expression of the gene for $\beta$ tubulin TUB2 is also reported, and in this case no increase of expression was seen in the wild-type, whereas increased expression in the auxin mutants was clearly apparent. Subsequently, the expression of two genes, i.e. CAT3 and FeSODI, considered involved in stress, and senescence was tested. The results, reported in fig. 2 , showed as in the wild-type there are clear variations in the expression, FeSOD/ being up-regulated at all the time lapses checked,

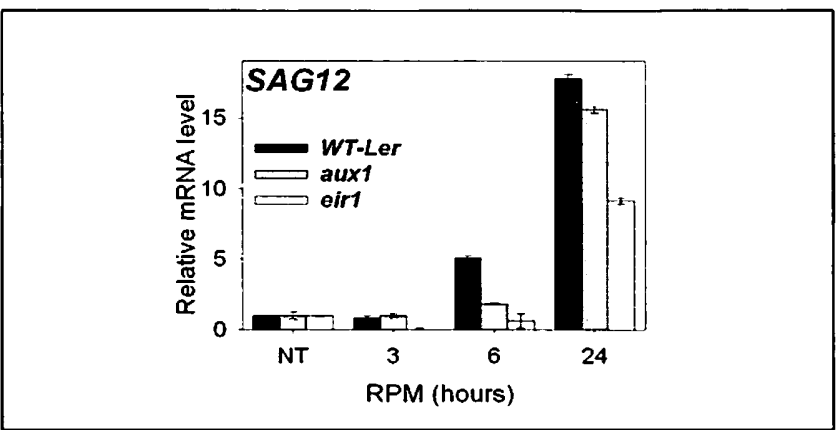

Figure 3. Microgrovity induced senescence in plants. Relative mRNA transcript of genes bound to the senescence (SAGI2) on wild-type Ler, aux $/$ and eirl/pin2 plants

whereas the expression of CAT3 was weakly downregulated. By contrast, in the mutants was seen only a weak up-regulation of FeSODI, whereas the data were scarcely significant for CAT3.Thereafter, the state of senescence of the plant was studied, considering that increase of senescence was frequently reported in organisms subject to space microgravity conditions. This was done by studying the expression of a gene that is considered connected with senescence, $S A G / 2$, and its expression appeared increased after 6 and 24 hours, with more evidence however in the wild-type (fig. 3).

\section{Discussion}

Under simulated microgravity conditions, we found a significantly difference when the transcription of TUA4 and TUA6 genes was studied in the auxin transport mutants aux $I$ and eir//pin2 with respect to the wild-type. In the wild-type in fact, the expression of TUA4 andTUA6 showed a peak after $3 \mathrm{~h}$ of microgravity, whereas in the mutants the peak was apparent after $6 \mathrm{~h}$. This result could be an indication that activation of tubulin expression may be involved in the detection of the gravity signal in plant, and is involved in controlling auxin transport. What is apparent, in fact, is that the disturbances in the transport of auxin in the mutants induce significant variations in the expression of the genes TUA4 and TUA6 after $6 \mathrm{~h}$. The increase in expression of FeSODI seen in the wild type, but not in the mutants (fig. 2) seems a sign of response of the plants to the stressing condition, which should have some connection with auxin regulated processes.A specific objective of our research was then, analyzing the expression of the gene target of senescence SAGI2, since it is already well known that microgravity and the space environment induce earlier aging [10]. In this part of the research what was found (fig. 3) is that the gene specifically involved in senescence SAGI2 [11] was up-regulated in the wild-type after $3 \mathrm{~h}$. In the case, however of aux / and eir//pin2, SAG / 2 was up-regulated after 24h.The altered transport of auxin thus delayed the expression of the gene and possibly the induction of senescence.

\section{Acknowledgements}

We thank the Italian Space Agency (ASI) for financial support. 
Alterations in the expression of genes for tubulin and stress in two Arabidopsis auxin mutants during simulated microgravity

\section{References}

[1] Wasteneys G.O. 2004. Progress in understanding the role of microtubules in plant cells. Curr. Opin. Plant Biol., 7: 651-660.

[2] Bisgrove S.R., Lee Y.R., Liu B., Peters N.T., Kropf D.L. 2008. The microtubule plus-end binding protein EB1 functions in root responses to touch and gravity signals in Arabidopsis. Plant Cell., 2: 396-410.

[3] Staiger C.J. 2000. Signaling to the actin cytoskeleton in plants. Annu. Rev. Plant Physiol. Plant Mol. Biol., 51: 257-288.

[4] Kalinina I. 2008. Microtubules spatial alterations in root cells of Brassica rapa under clinorotation. Cell. Biol. Int., 5: 581-583.

[5] Baluska F, Hasenstein K.H. 1997. Root cytoskeleton: its role in perception of and response to gravity. Planta, 203(Suppl): S6978.

[6] Migliaccio F, Piconese S. 2001. Spiralizations and tropisms in Arabidopsis roots. Trends Plant Sci., 12: 561-565.
[7] Piconese S., Tronelli G., Pippia P., Migliaccio F. 2003. Chiral and non-chiral nutations in Arabidopsis roots grown on the random positioning machine. J. Exp. Bot., 389: 1909-1918.

[8] Migliaccio F., Fortunati A., Tassone P. 2009. Arabidopsis root growth movement and their symmetry. Plant Signal. Behav., 4: 183-190.

[9] Ottenschläger I.,Wolff P.,Wolverton C., Bhalerao R.P., Sandberg G., Ishikawa H., Evans M., Palme K. 2003. Gravityregulated differential auxin transport from columella to lateral root cap cells. Proc. Natl. Acad. Sci. U.S.A., 100: 2987-2991.

[10] Biolo G., Heer M., Narici M., Strollo F. 2003. Microgravity as a model of aging. Curr. Opin. Clin. Nutr. Metab., 6: 31-40.

[11] Wagstaff C., Yang T.J., Stead A.D., Buchanan-Wollaston V., Roberts J.A. 2009.A molecular and structural characterization of senescing Arabidopsis siliques and comparison of transcriptional profiles with senescing petals and leaves. Plant J., 57:690-705. 